\title{
Fatal Cerebral Haemorrhage in a Thrombolysed Patient with Ischaemic Stroke Who Developed Interval Thrombocytopaenia from Acute Dengue Infection
}

\section{Dear Editor,}

Intravenous thrombolysis is an evidence-based treatment in acute ischaemic stroke. Symptomatic intracranial haemorrhage $(\mathrm{sICH})$ is a known complication of intravenous thrombolysis with a prevalence rate of $5-6 \% .{ }^{1}$ The known predictors of sICH include high blood pressure during thrombolysis, ${ }^{2}$ large volume of ischaemic change on imaging studies, ${ }^{3}$ extensive cerebral microbleeds ${ }^{4}$ and extensive leukoariosis. ${ }^{5}$ We report a rare case of devastating $\mathrm{sICH}$ following thrombolysis for ischaemic stroke with underlying thrombocytopaenia that was attributed to dengue fever.

\section{Case Presentation}

A 73-year-old man with a history of hypertension and hyperlipidaemia - managed with Niften (slow-release
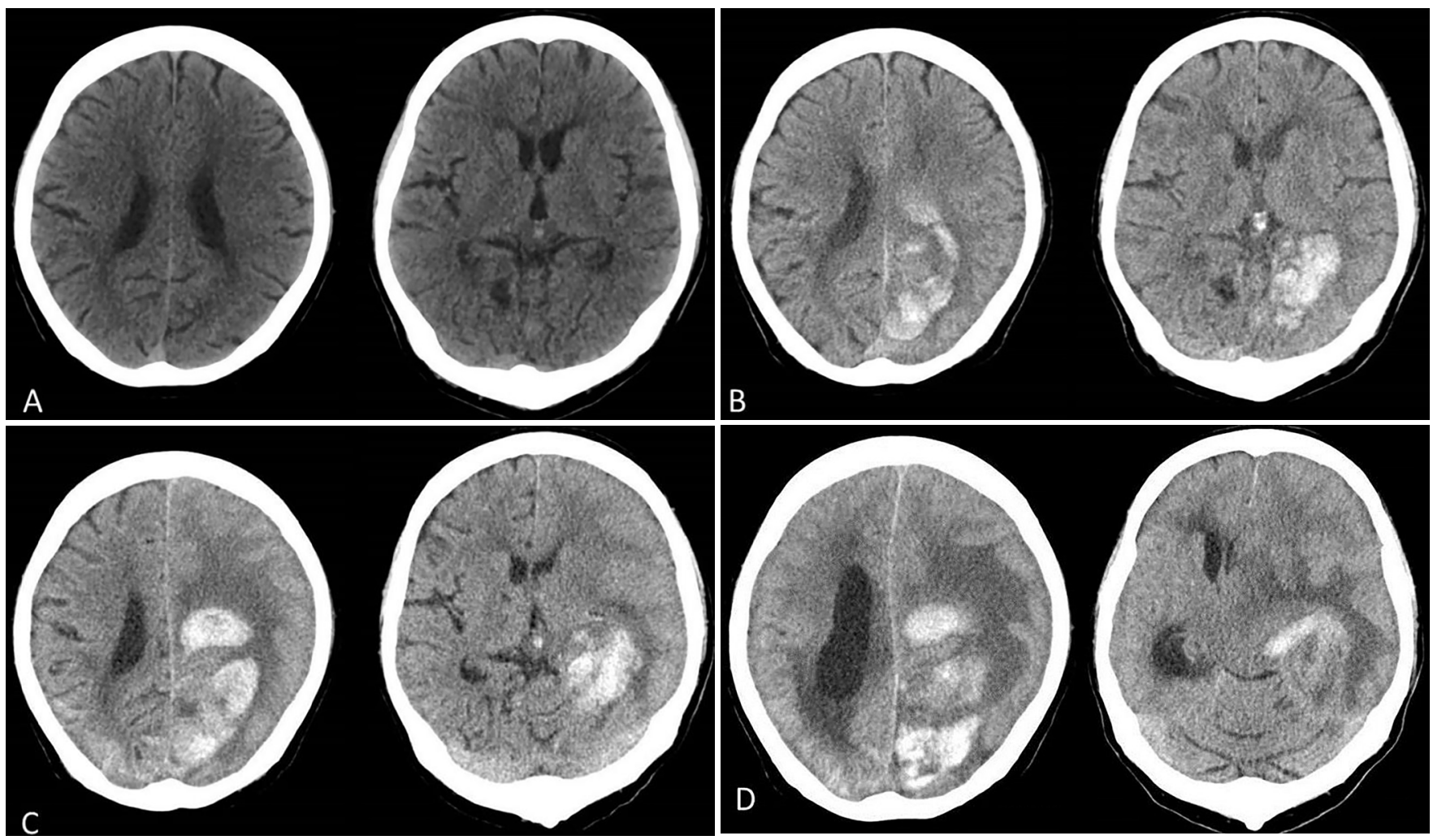

Fig. 1. Computed tomography of brain at (A) pre-thrombolysis, (B) 8 hours post-thrombolysis, (C) day 9 and (D) day 16 of admission. 


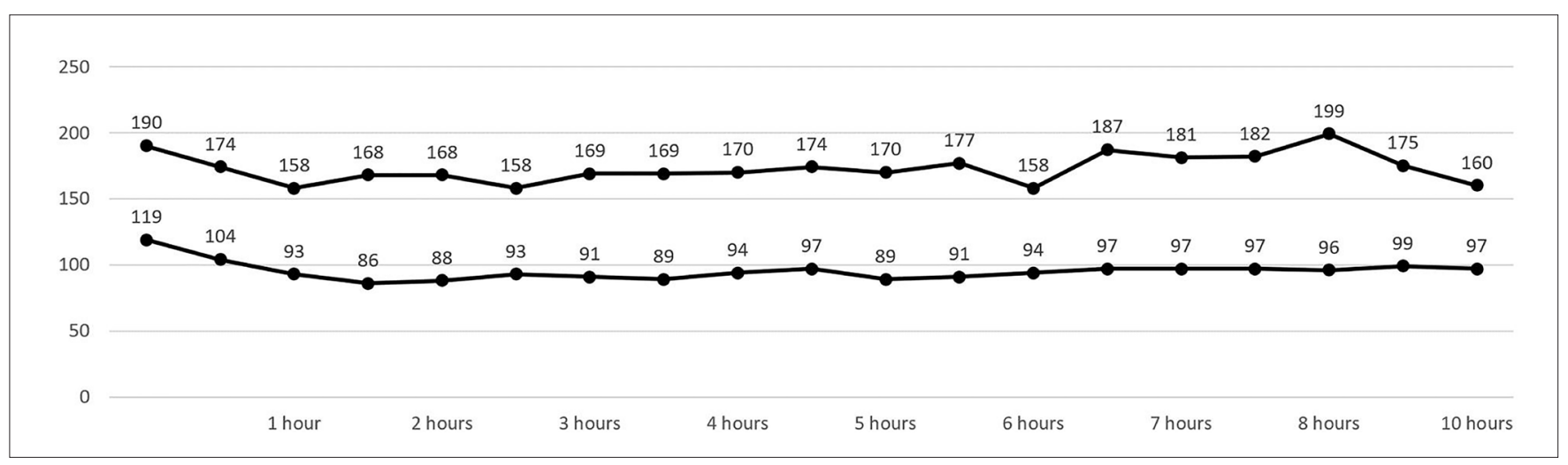

Fig. 2. Non-invasive blood pressure ( $\mathrm{mm} \mathrm{Hg}$ ) levels up to 10 hours post-thrombolysis. Upper line denotes systolic blood pressure and lower line denotes diastolic blood pressure.

He was treated with standard intravenous alteplase dose of $0.9 \mathrm{mg} / \mathrm{kg}$ which was initiated 4 hours after symptom onset. Over the next 8 hours, his blood pressure ranged between 150/80-160/90 $\mathrm{mmHg}$. At 2 hours post-thrombolysis, an isolated reading of $190 / 120 \mathrm{mmHg}$ fell to $158 / 93 \mathrm{mmHg}$ after 30 minutes (Fig. 2), the signs and symptoms resolved and NIHSS score was nil.

At 8 hours post-thrombolysis, he developed headache. Physical examination revealed right homonymous hemianopia. Brain $\mathrm{CT}$ showed left parieto-occipital parenchymal haemorrhage 1 (PH1) (Fig. 1B) according to the definition of Safe Implementation of Treatments in Stroke. ${ }^{6}$ A new area of hypodensity was seen over the right thalamic region that was suggestive of an evolving infarct, and there was a spike in blood pressure to 199/96 $\mathrm{mmHg}$ (Fig. 2). Transdermal glyceryl trinitrate patch $2.5 \mathrm{mg}$ and oral amlodipine $2.5 \mathrm{mg}$ were administered intermittently to manage blood pressure. Laboratory tests showed normal full blood count (haemoglobin $15.5 \mathrm{~g} / \mathrm{dL}$, total white blood cell [WBC] count $12 \times$ $10^{9} / \mathrm{L}$ and platelet $377 \times 10^{9} / \mathrm{L}$ ), coagulation profile (activated partial thromboplastin time of 30 seconds and thromboplastin time of 16.2 seconds), serum fibrinogen level $(0.46 \mathrm{~g} / \mathrm{L})$ and liver function. He was treated with 6 units of cryoprecipitate concentration.

On day 2, he was started on oral amlodipine $5 \mathrm{mg}$ once daily and repeat brain CT showed stable size of left parieto-occipital haemorrhage. On day 3, magnetic resonance image (MRI) of the brain showed multiple foci of acute infarction over both cerebellar lobes and established infarction of right thalamus (Fig. 3). Magnetic resonance angiography did not show significant intracranial large artery stenosis, and signs of aneurysm or cerebral microbleeds were absent. The diagnosis was acute right thalamic infarction and haemorrhagic transformation of silent left occipital infarct of grade PH1. Echocardiography and electrocardiogram monitoring did not detect the source of cardioembolism.

Between day 4 and day 8 , he had daily temperature spikes (Fig. 4) and his blood pressure ranged between $150 / 80-170 / 100 \mathrm{mmHg}$. His neurological status was stable with no new physical signs and his headache improved with analgesia. Chest radiograph, blood and urine culture findings were normal, and he was treated as presumptive viral fever. WBC count ranged between $8 \times 10^{9} / \mathrm{L}-10 \times 10^{9} / \mathrm{L}$ (Fig. 5). In view of intracranial haemorrhage, antiplatelet therapy was not started.

On day 8 , the fever settled. Full blood count taken on the same day revealed thrombocytopaenia of 11 $\times 10^{9} / \mathrm{L}$ (Fig. 5), a marked drop from the normal platelet level seen on day 4 . There was a concurrent raise in total WBC count of $20 \times 10^{9} / \mathrm{L}$ (Fig. 6) with monocytosis $\left(1.2 \times 10^{9} / \mathrm{L}\right)$ and serum transaminases

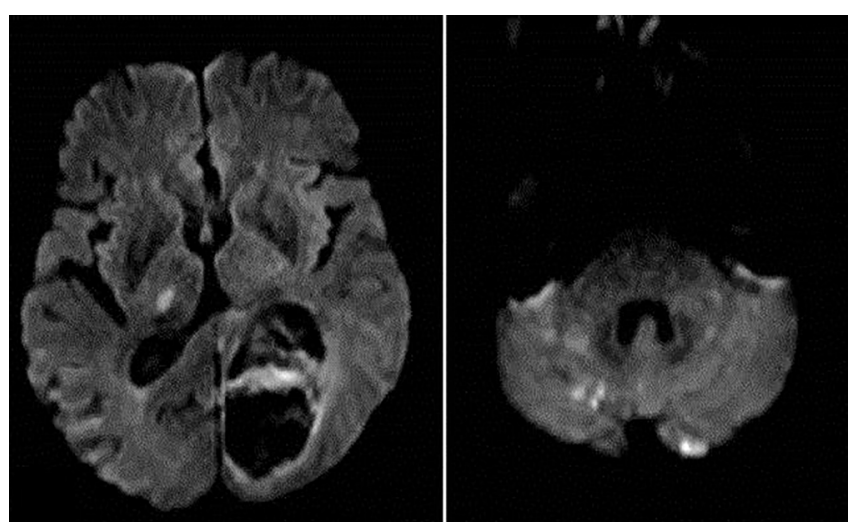

Fig. 3. Diffusion-weighted magnetic resonance image of brain on day 3 of admission. 


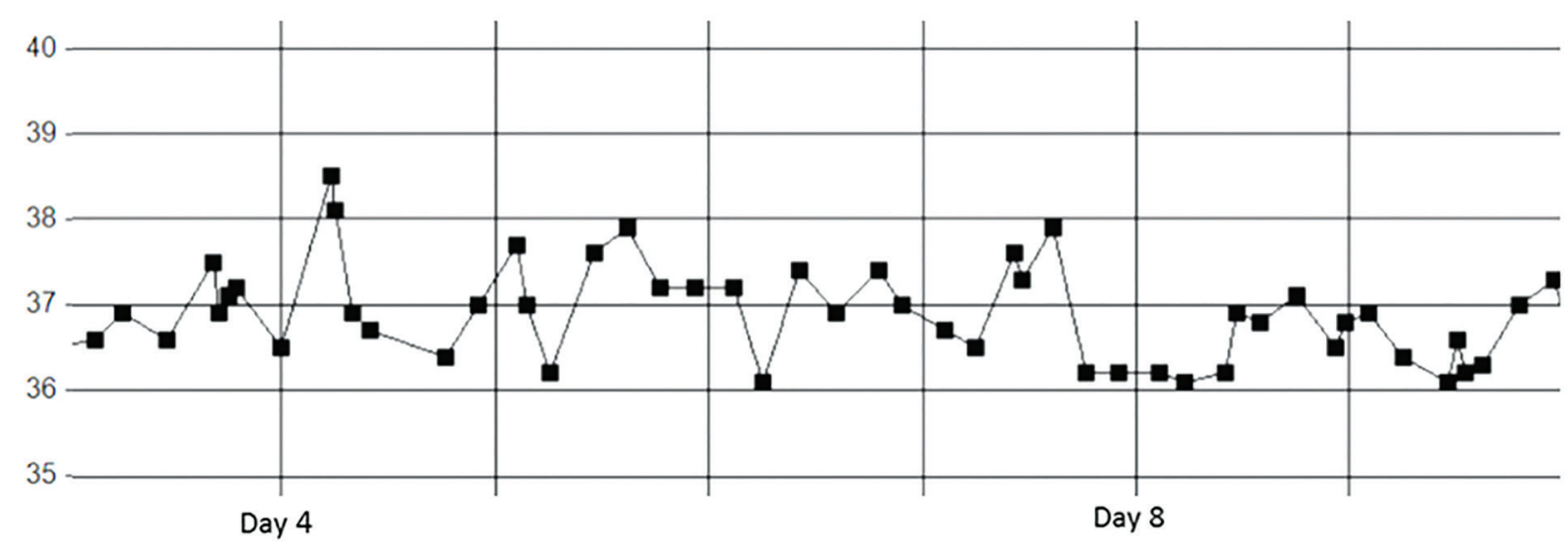

Fig. 4. Temperature (degree Celsius) readings from day 4 to day 10.

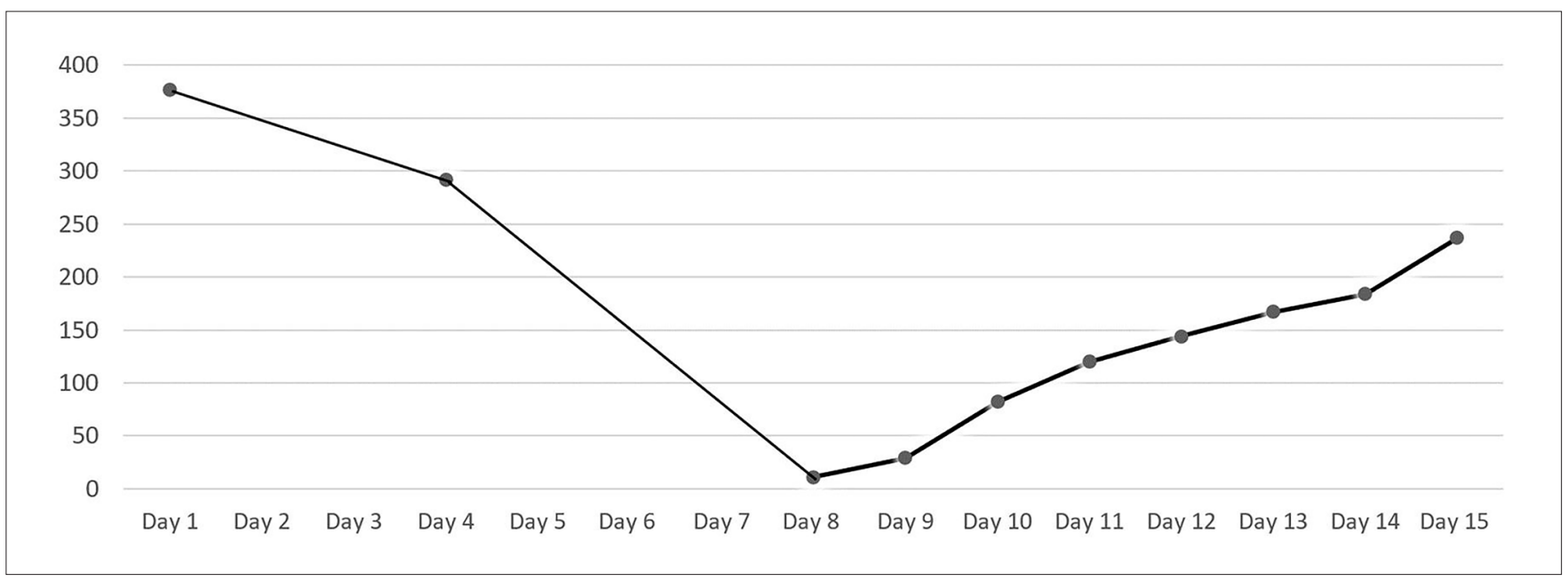

Fig. 5. Platelet count $\left(\times 10^{9} / \mathrm{L}\right)$ from day 1 to day 15 .

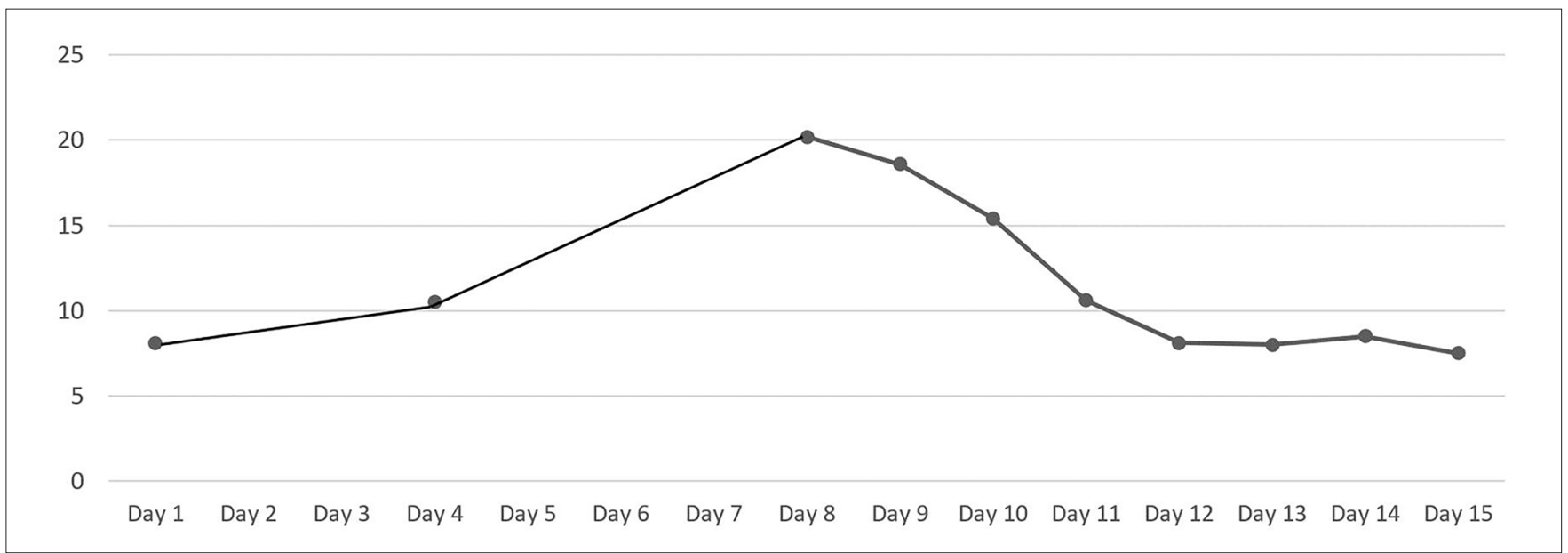

Fig. 6. White blood cell count $\left(\times 10^{9} / \mathrm{L}\right)$ from day 1 to day 15 . 
(alanine aminotransferase $178 \mathrm{U} / \mathrm{L}$ and aspartate aminotransferase $233 \mathrm{U} / \mathrm{L}$ ). Coagulation profile and serum fibrinogen level remained normal. Reversetranscription polymerase chain reaction tested positive for dengue virus ribonucleic acid, but dengue serotyping was not performed. Physical examination did not show ascites, hepatosplenomegaly, petechial rash or pleural effusion. One unit of platelet concentrate was transfused; at 10 hours post-transfusion, repeat platelet count showed a reading of $27 \times 10^{9} / \mathrm{L}$. Patient remained clinically stable and afebrile.

On day 9, repeat brain CT showed extension of left cortical haemorrhage, but no associated mass effect (Fig. 1D). Platelet count was $48 \times 10^{9} / \mathrm{L}$. Another unit of platelet concentrate was administered and it was gradually increased from $29 \times 10^{9} / \mathrm{L}$ to $48 \times 10^{9} / \mathrm{L}$, $82 \times 10^{\%} / \mathrm{L}$ and $129 \times 10^{9} / \mathrm{L}$ (Fig. 4). However, right hemiparesis and aphasia worsened progressively. On day 16, brain CT showed progression from $\mathrm{PH} 1$ to $\mathrm{PH} 2$ (Fig. 1E). The level of consciousness dropped rapidly, and he died on the same day.

\section{Discussion}

This is the first reported case of sICH following stroke thrombolysis with dengue fever. Dengue is a vector-borne disease caused by a flavivirus transmitted by the Aedes aegypti mosquito. Previous case reports on dengue that contributed to ischaemic stroke ${ }^{7-9}$ had reported onset of ischaemic stroke during the febrile stage of dengue which ranged between day $1-15$ of fever. A few reports suggested that thrombotic risk in dengue fever can be heightened by factors such as increased levels of immunoglobulin M against phospholipids ${ }^{10}$ and lupus anticoagulants, ${ }^{11}$ increased plasminogen activator inhibitor-1 plasma levels, low concentrations of plasma anticoagulant proteins $\mathrm{C}$ and $\mathrm{S}$ and antithrombin III, and disseminated intravascular coagulopathy. It was proposed that inflammation could be attributed to infections that cause hypercoagulable state. ${ }^{12-13}$

In our patient, we postulate that dengue did not contribute to ischaemic stroke since clinical features of dengue or thrombocytopaenia at initial stroke presentation were absent. The fever started 4 days after the onset of ischaemic stroke, but the aetiology of ischaemic stroke could not be determined. Findings from initial investigations did not show cardioembolism, large vessel obstruction and infective endocarditis. Coagulation profile screen was also normal.

Primary intracranial haemorrhage with dengue fever has been reported in a few case studies ${ }^{14-16}$ that cited coagulopathy, platelet dysfunction, thrombocytopaenia and vasculopathy as possible causes. Bleeding from dengue infection is also attributed to direct fibrinolysis effect by dengue virus. ${ }^{17}$

Our patient had parenchymal haemorrhage (PH) of asymptomatic infarct due to thrombolysis. He remained stable for 4 days after onset of $\mathrm{PH}$, but subsequently deteriorated with worsening hemiparesis and progressive drowsiness. CT findings also showed progression from $\mathrm{PH} 1$ to $\mathrm{PH} 2$ with associated mass effect. $\mathrm{PH}$ progression may be explained by severe thrombocytopaenia associated with dengue haemorrhagic fever and, possibly, endothelial leakage which is known to occur with dengue infection.

During the febrile phase of dengue fever, an immunemediated process involving inflammatory cytokinessuch as monocyte chemo-attractive protein-1-has been observed to cause alteration of tight junction of vascular endothelium leading to plasma leakage. Other cytokines - such as certain interleukins and platelet-derived growth factors - may lead to platelet destruction and limit platelet aggregation that may precede the defervescence phase. ${ }^{18}$

\section{REFERENCES}

1. Seet RCS, Rabinstein AA. Symptomatic intracranial hemorrhage following intravenous thrombolysis for acute ischemic stroke: a critical review of case definitions. Cerebrovasc Dis 2012;34:106-14.

2. Bluhmki E, Chamorro A, Dávalos A, Machnig T, Sauce C, Wahlgren $\mathrm{N}$, et al. Stroke treatment with alteplase given $3.0-4.5 \mathrm{~h}$ after onset of acute ischaemic stroke (ECASS III): additional outcomes and subgroup analysis of a randomised controlled trial. Lancet Neurol 2009;8:1095-102.

3. Larrue V, von Kummer R, Müller A, Bluhmki E. Risk factors for severe hemorrhagic transformation in ischemic stroke patients treated with recombinant tissue plasminogen activator: a secondary analysis of the European-Australasian Acute Stroke Study (ECASS II). Stroke 2001;32:438-41.

4. Dannenberg S, Scheitz JF, Rozanski M, Erdur H, Brunecker P, Werring DJ, et al. Number of cerebral microbleeds and risk of intracranial hemorrhage after intravenous thrombolysis. Stroke 2014;45:2900-5.

5. Kongbunkiat K, Wilson D, Kasemsap N, Tiamkao S, Jichi F, Palumbo $\mathrm{V}$, et al. Leukoaraiosis, intracerebral hemorrhage, and functional outcome after acute stroke thrombolysis. Neurology 2017;88:638-45.

6. SITS International. Thrombolysis Registry. Available at: http:// www.sitsinternational.org/registries/sits-thrombolysis/. Accessed on 1 June 2019

7. Verma R, Sahu R, Singh AS, Atam V. Dengue infection presenting as ischemic stroke: an uncommon neurological manifestation. Neurol India 2013;61:317-8.

8. Liou LM, Lan SH, Lai CL. Dengue fever with ischemic stroke: a case report. Neurologist 2008;14:40-2.

9. Mathew S, Pandian JD. Stroke in patients with dengue. J Stroke Cerebrovasc Dis 2010;19:253-6.

10. da Costa PSG, Ribeiro GM, Soares Jr C, da Costa Campos L. Severe thrombotic events associated with dengue fever, Brazil. Am J Trop Med Hyg 2012;87:741-2. 
11. Chen WH. An unusual transitory increase of lupus anticoagulant in dengue virus infection complicated with cerebral ischaemia. J Infect 2006;52:e87-91.

12. Lin CF, Wan SW, Cheng HJ, Lei HY, Lin YS. Autoimmune pathogenesis in dengue virus infection. Viral Immunol 2006;19:127-32.

13. Esmon CT. Inflammation and thrombosis. J Thromb Haemost 2003;1:1343-8.

14. Sam JE, Gee TS, Nasser AW. Deadly intracranial bleed in patients with dengue fever: a series of nine patients and review of literature. J Neurosci Rural Pract 2016;7:423-34.

15. Vargas-Sánchez A, Chiquete E, Gutiérrez-Plascencia P, CastañedaMoreno V, Alfaro-Castellanos D, Paredes-Casillas P, et al. Cerebellar hemorrhage in a patient during the convalescent phase of dengue fever. J Stroke 2014;16:202-4.

16. Kumar R, Prakash O, Sharma BS. Intracranial hemorrhage in dengue fever: management and outcome: a series of 5 cases and review of literature. Surg Neurol 2009;72:429-33.

17. Kurukularatne C, Dimatatac F, Teo DLT, Lye DC, Leo YS. When less is more: can we abandon prophylactic platelet transfusion in dengue fever? Ann Acad Med Singapore 2011;40:539-45.
18. Rathakrishnan A, Wang SM, Hu Y, Khan AM, Ponnampalavanar S, Chai LSL, et al. Cytokine expression profile of dengue patients at different phases of illness. PLoS One 2012;7: e52215.

Yee Hau Pang, ${ }^{1}$ MRCP, Tushar Gosavi Divakar, ${ }^{1}$ MRCP, Deidre Anne De Silva, ${ }_{F R C P}$

${ }^{1}$ Department of Neurology, National Neuroscience Institute (Singapore General Hospital Campus), Singapore

Address for Correspondence: Dr Pang Yee Hau, Department of Neurology, National Neuroscience Institute (Singapore General Hospital Campus), Outram Road, Singapore 169608.

Email: pang.yee.hau@singhealth.com.sg 